\title{
Macro-texture perception through active and dynamic touch
}

\author{
Wenjing Chen ${ }^{1,2}$, Nan $\mathrm{Li}^{1}$, Liangzong $\mathrm{He}^{1}$, Lantao Huang ${ }^{1}$ and Tao Zeng ${ }^{1,2, a}$ \\ ${ }^{1}$ Dept. of Instrumental and Electrical Engineering, Xiamen University, Xiamen, 361005, China \\ ${ }^{2}$ State Key Laboratory of Virtual Reality Technology and Systems, Beihang University, Beijing 100191, China
}

\begin{abstract}
The tactile mechanism of texture perception is getting well figured out, resulting in the emergence of texture rendering devices. It is worth noting that the textures in these studies have interior space gap in the millimeter level and below. This kind of textures is called "fine texture". In contrast, the "Macro texture" has space gap in centimeters and above, and it is considered as the special texture with tiny texture pattern covered on curved surface. Compared with fine texture, macro texture contains richer surface information, including both roughness and curvature. This paper aims to study the mechanism of macro texture perception, and establish active and dynamic touch-based haptic perception models.
\end{abstract}

Keywords: macro texture perception; curvature perception; active; dynamic touch.

\section{Introduction}

Haptic perception of texture is well studied over recent years. The display methods could be divided into three categories: the first is based on geometric shape reproduce, it gets texture's geometric contour through image processing, and then uses force feedback device to display contact force normally and tangentially [1]; the second is based on vibration perception, the researchers constructed array of needles and controlled their vibration amplitude and frequency to reproduce the texture [2-4]; the third is based on the friction force change, it could get texture feeling by changing the contact friction between the finger and the interface of haptic device, like Tesla Touch [5] and StimTac [6].

The common characteristic of the rendered texture using above methods is that their interior space gap is in the millimeter and below. We could call them "fine texture". On contract, the "macro texture” has interior space gap in centimeter and above (Fig. 1a). From the point of view of geometric properties, the macro texture can be seen as tiny texture pattern (Fig. 1b) covering on curved surface (Fig. 1c), it includes both texture and curvature information. Human could get both the feeling of the roughness of fine texture and the feeling of force feedback of the curvature surface when exploring across a macro texture.

Compared with fine texture, the macro texture contains richer information and its haptic mechanism is certainly more complex. For example, the deformation of fingertip's tissue, the force applied by the finger, the shearing force fed back by the surface, the friction force between the finger and the surface, etc, are different from that of the fine texture exploration. These differences will change the characteristic value and passivity boundary condition of the common fine texture perception models. In addition, from the view of curvature perception, thanks to the tiny texture pattern coved on the

\footnotetext{
${ }^{a}$ Corresponding author : tao.zeng@xmu.edu.cn
} 
surface, the curvature display should render not only the geometrical property and force feedback [7, 8], but also the micro mechanical stimuli between the skin and the rough surface.

This work studies the haptic mechanism of the macro texture perception, and establishes rendering models which are suited to most macro textures. These models should take consider of the interplay between fine texture perception and curvature perception. The paper is organized as follows: Section 2 introduces the perception protocol of macro texture; Section 3 describes the motion models of the kinesthetic platform; Section 4 presents the analysis of fine texture rendering; and Section 5 gives the conclusion and future work.

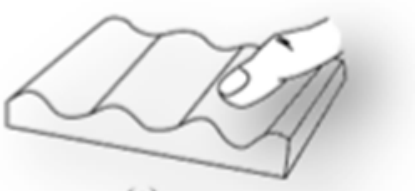

(a)
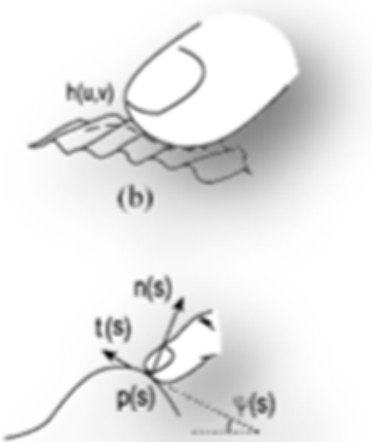

(c)

Figure 1. The haptic perception of macro texture. (a) The finger explores the macro texture; (b) The finger tip gets in touch with the fine texture pattern; (c) The finger-tip percepts the curvature.

\section{Perception prototype}

The perception of macro texture can be hardly achieved by using neither existed fine texture displays nor force feedback devices. For instance, the needle array-based or friction change-based displays could only reproduce the sensation of roughness with low amplitude, but not the texture grid with high amplitude [2]. The force feedback device, such as the PhanToM, could reconstruct the contour of the macro texture, but cannot display the tiny texture pattern. At the same time, its instability and small force output limits the application when rigid contact existed [9].

In consideration of the perception of fine texture and curvature simultaneously, the StimTac plate is used as the end-effector [10] (Fig. 2). It's hard and successive surface could display the fine texture based on film squeeze effect, and the curvature based on the reproduction of local contact trajectory. The prototype and picture of apparatus are shown in Fig. 3. It has three degrees of freedom: x-axis, zaxis and rotation about y-axis. It can independently orient, elevate, and translate the StimTac plate, making sure that the plate is always kept tangential to a virtual macro texture at the contact point during manual exploration.

This platform can meet the requirement of active and dynamic touch. The finger of the user is free to move forward and backward along the surface of the plate. It is closer to natural touch and at the same time, it is in regard to slip cue over the surface [11].According to the characteristic of macro texture and the prototype of apparatus, three motion control models are built, in making sure that they meet the needs of continuity on the force response, conservative and passivity on energy. 


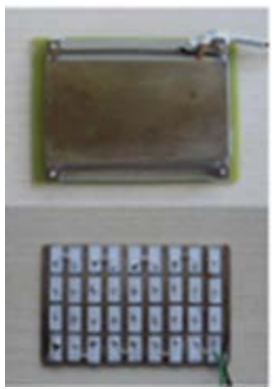

(a)

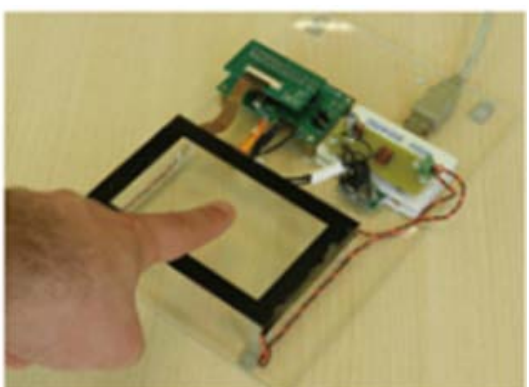

(b)

Figure 2. The StimTac plates.(a) is metal surface prototype, and (b) is transparent capacitive prototype [10].

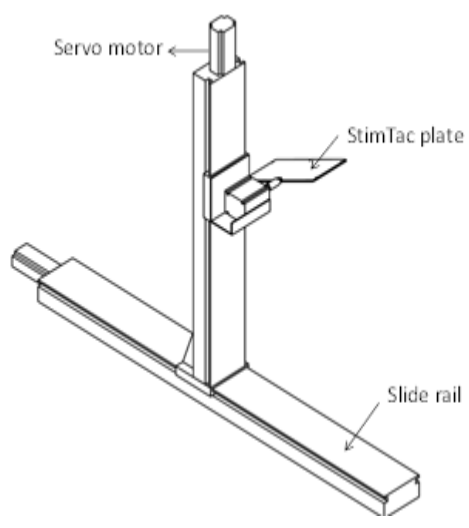

(a)

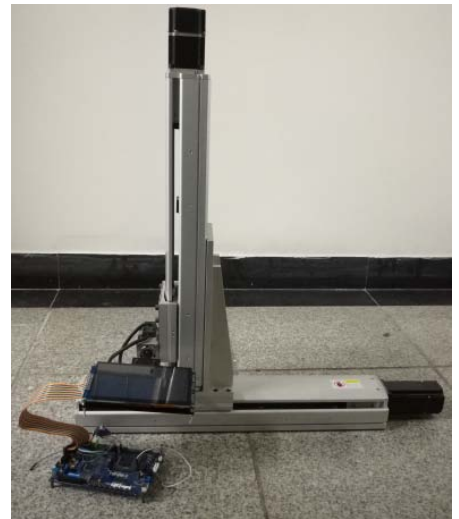

(b)

Figure 3. (a) is the prototype; (b) is the photo of apparatus.

\section{Analysis of the motion models}

For position control of the end effector, three motion models (control strategies) were proposed. When user's finger moves forward and backward along the surface of the StimTac plate freely, the plate rotates about its midpoint to make sure that it is always tangential to the profile of the macro texture to be represented. This control strategy was applied to respect the dominant information for the shape perception - the local surface orientation $[12,13]$. As the end effector rotates itself, velocity of contact point at the plate is existed. How to counterbalance that velocity leads to the second motion model. Moreover, thanks to the slipping movement between the finger and the plate, a slip cue exists during the exploration. How to respect this slip cue leads to the third motion model.

\subsection{Basic motion model}

The sinusoidal profile with one degree of freedom was used to describe the contour of macro texture (see Fig. 4). In order to haptic display this profile, we control the orientation of the end effector in a sinusoidal way so that the plate is always tangential to the profile of the macro texture at the contact point, when exploration takes place following the proximal-distal direction. 


\section{AEST2016}
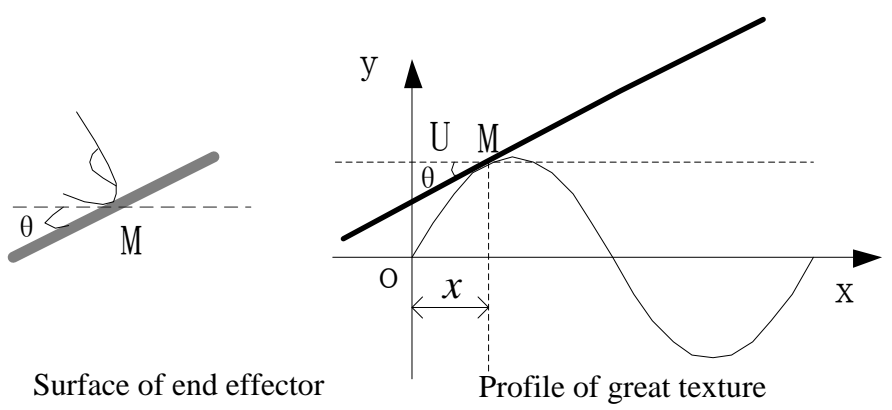

Surface of end effector

(a)

(b)

Figure 4. Contact schematic on macro texture.

Fig. 4 shows the rendering process. $M$ is the contact point; $U$ is the length of exploration distance; $\theta$ is the angle between the finger and the tactile plate at the contact point, it defines the cue of local surface orientation.

The sinusoidal profile of macro textures is:

$$
y(x)=A \sin \left(\frac{2 \pi x}{T}\right)
$$

Relationship between $\theta$ and $\mathrm{x}$ is:

$$
\tan \theta=\frac{\mathrm{dy}(\mathrm{x})}{\mathrm{dx}}=\frac{2 \pi \mathrm{A}}{\mathrm{T}} \cos \left(\frac{2 \pi \mathrm{x}}{\mathrm{T}}\right)
$$

Relationship between $\mathrm{U}$ and $\mathrm{x}$ is:

$$
\mathrm{U}=\int_{0}^{\mathrm{x}} \sqrt{1+\frac{4 \pi \AA}{\tau} \mathrm{T}^{2} \pi \tau} \cos ^{2}\left(\frac{2}{\mathrm{~T}}\right) d
$$

Where $T$ and $A$ are the period and amplitude of the macro texture respectively. According to (1) (2) (3), we can obtain the relationship between the angle $\theta$ and finger exploration distance $U$ at the sinusoidal macro texture model.

\subsection{Velocity compensation model}

As we know, human exploration takes place mainly in the condition that the touched object is static, and the finger is dynamic (self-controlled touch, leads to a better performance than other manners of touch). Nevertheless, the end effector of above platform has its own velocity during haptic exploration. Fig. 5 shows the tactile plate's rotation movement. From the time $t_{1} t_{2} t_{2}$, the initial contact point $M_{1}$ moves to the position $M_{2}$ ' (at the instant $t_{2}$, the contact point is $M_{2}$ ). So the contact point $M_{1}$ has the velocity $v_{x}$ at $\mathrm{x}$-direction and $v_{y}$ at y-direction as the plate rotates.

The two velocity components could be calculated from the exploration speed and the parameters of macro texture. Define: the length of the tactile plate is $L$, and then the rotation angular velocity of the plate is $\omega$. According to the relationship between the angular velocity and linear velocity, we can get the speed of the contact point $V$ : 


\section{AEST2016}

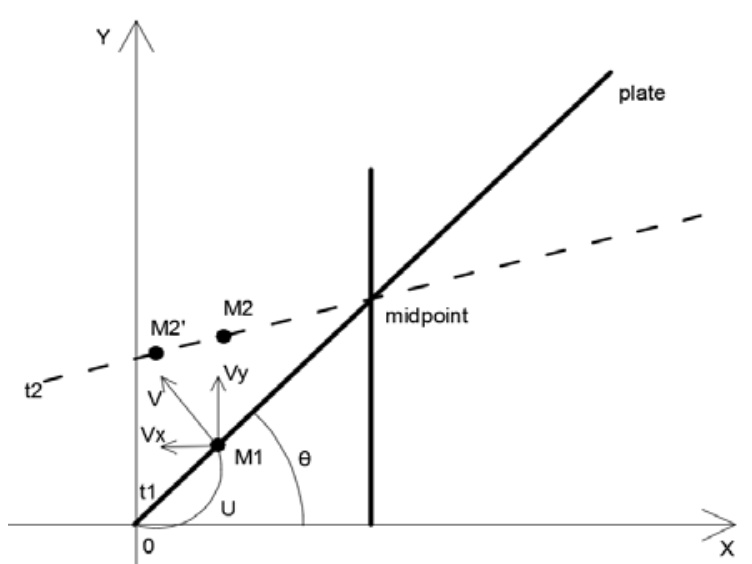

Figure 5. Illustration of the contact point's velocity during finger exploration.

When $\mathrm{U}<\mathrm{L} / 2$,

$$
V=\left(\frac{L}{2}-U\right) \omega
$$

When $\mathrm{U}>\mathrm{L} / 2$,

$$
V=\left(U-\frac{L}{2}\right) \omega
$$

As shown in Fig.5, we decompose the speed v into two components at horizontal and vertical directions:

$$
\begin{aligned}
& V_{x}=V \sin \theta \\
& V_{y}=V \cos \theta
\end{aligned}
$$

In order to restore the real situation, it is necessary to compensate that velocity of contact point. The strategy is to add two degrees of freedom to the platform, making the plate has opposite velocity against the two components $v_{x}$ and $v_{y}$. According to (6) (7) and the midpoint of the tactile plate, the compensation values and directions were obtained, as shown in Table 1.

Table 1.The Value and Direction of Velocity Compensation

\begin{tabular}{l|l|l|l|l|l|l|l|l}
\hline \multirow{2}{*}{} & \multicolumn{3}{|c|}{ Left of midpoint } & \multicolumn{4}{c}{ Right of modpoint } \\
\cline { 2 - 9 } & $\begin{array}{l}\text { Horizontal direc- } \\
\text { tion }\end{array}$ & Vertical direction & \multicolumn{3}{l}{$\begin{array}{l}\text { Horizontal direc- } \\
\text { tion }\end{array}$} & \multicolumn{2}{l}{ Vertical direction } \\
\cline { 2 - 9 } & value & Dirrection & value & Dirrection & value & Dirrection & value & Dirrection \\
\hline & & right & & down & & left & Vcos $\theta$ & up \\
\hline & & left & Vcos $\theta$ & down & & right & & up \\
\hline & & right & & up & & lest & Vcos $\theta$ & down \\
\hline & & left & V $\cos \theta$ & up & & right & & down \\
\hline
\end{tabular}




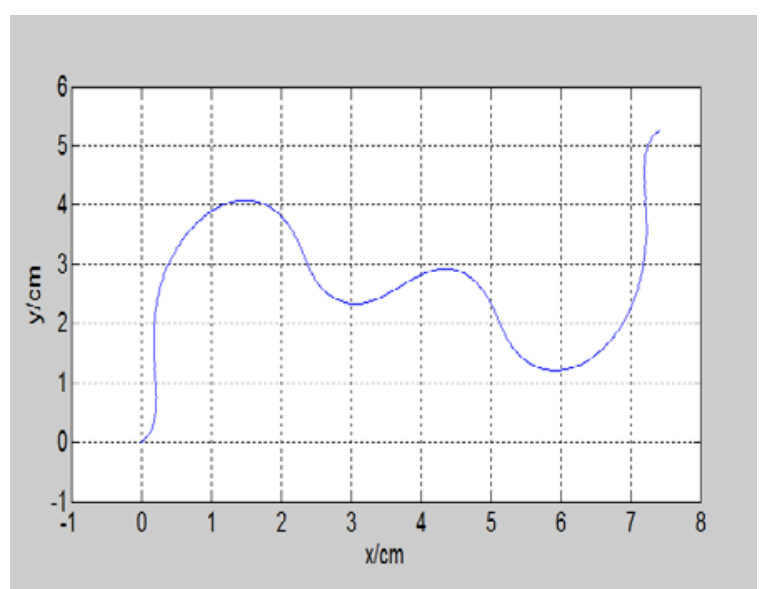

Figure 6. The exploration trajectory of the fingertip (Period $=4 \mathrm{~cm}$, Amplitude $=0.5 \mathrm{~cm})$.

\subsection{Position compensation model}

For each contact point $M$, the basic motion model has a corresponding angle $\theta$. The exploration distance $U$ can be got based on $\theta$ and $M$. Fig. 6 presents the exploration trajectory based on x-ray. It is worth noting that the curve of exploration trajectory is notable different from the profile of macro texture. This difference may influence the perception of curvature.

Position compensation was then proposed to coincide the exploration trajectory with the profile of macro texture. The strategy is that, the flat plate was displaced in the $\mathrm{x}$-axis direction and the $\mathrm{y}$-axis direction to compensate the redundant displacement compared with the profile of macro texture. At the same time, the rotation angle $\theta$ is strictly guaranteed as calculated in (2), so the dominant slop cue for curvature perception is always respected. Thus, the motion model has three degrees of freedom: rotation DOF, horizontal DOF (displacement in x-axis direction) and vertical DOF (displacement in yaxis direction).Define the coordinate system of the macro texture model has the left end point as the origin point and the initial angle of the platform is $\theta_{0}$, the additional displacement $x_{0}$ and displacement $y_{0}$ of the plate could be evaluated by the following equation:

When $\mathrm{U}<\mathrm{L} / 2$,

$$
\begin{aligned}
& x_{\text {finger }}=\frac{L}{2} \cos \theta_{0}-\left(\frac{L}{2}-U\right) \cos \theta \\
& y_{\text {finger }}=\frac{L}{2} \sin \theta_{0}-\left(\frac{L}{2}-U\right) \sin \theta
\end{aligned}
$$

When $\mathrm{U}>\mathrm{L} / 2$,

$$
\begin{gathered}
y_{\text {finger }}=\frac{L}{2} \sin \theta_{0}+\left(U-\frac{L}{2}\right) \sin \theta \\
x_{\text {finger }}=\frac{L}{2} \cos \theta_{0}+\left(U-\frac{L}{2}\right) \cos \theta \\
x_{0}=x_{\text {finger }}-x_{\text {real }} \\
y_{0}=y_{\text {finger }}-y_{\text {real }}
\end{gathered}
$$


Where, $\mathrm{x}_{\text {finger }}$ and $\mathrm{y}_{\text {finger }}$ are the horizontal coordinate and vertical coordinate of the finger in the coordinate system of the platform respectively, $\mathrm{x}_{\text {real }}$ and $\mathrm{y}_{\text {real }}$ are the horizontal coordinate and vertical coordinate of the point to be rendered in the coordinate system of the platform respectively, $L$ is the length of the tactile plate.

Fig. 7 and Fig. 8 present the Matlab simulation of the compensation displacements of $x_{0}$ and $y_{0}$.

\section{Fine texture pattern rendering}

As Fig. 1 shows, tiny texture pattern is covered on the profile of macro texture. The perception of tiny texture pattern and the perception of curvature could be realized independently. But they have strong internal relationship and better to be considered together. In that condition, the roughness and force are mixed, and the micro mechanical stimuli will be more complex. The deformation and the stress force of the fingertip's tissue are also more complex than either case. The tiny pattern could be rendered by tactile displays based on various principles. Comparatively, the displays with frictioncontrolled surface have better rendering results $[6,14,15]$. Among these devices, the StimTac has excellent fine texture perception effect. It is a touchpad device that supports friction reduction by means of squeeze film effect.

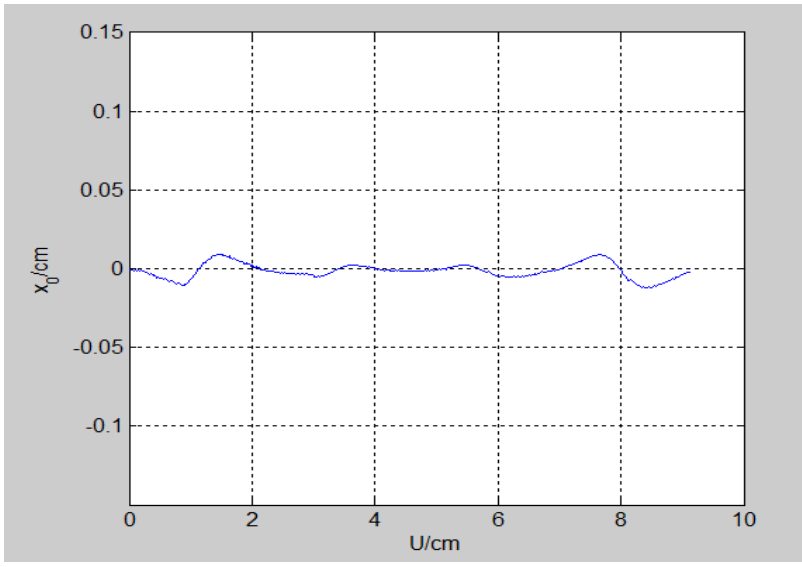

Figure 7. The compensation displacement of $\mathrm{x}_{0}$.

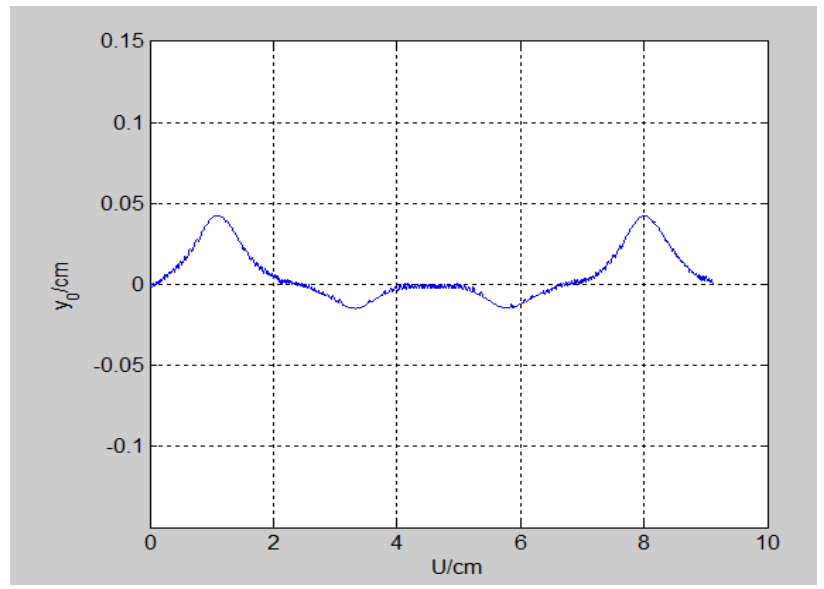

Figure 8. The compensation displacement of $\mathrm{y}_{0}$.

The creation of squeeze film effect is a particular technique for producing a variable friction between the finger and the touched surface. Variable friction force reproduces variable roughness. 
The StimTac has successive surface and the user's finger could explore it actively and dynamically at proximal-distal direction, so it is used as the end effector in this study for fine texture perception and curvature perception meanwhile.

\section{Conclusion and future work}

In this work, we studied the haptic perception of macro texture. The macro texture can be seen as tiny texture pattern covering on curved surface, it includes both fine texture and curvature information. Its haptic mechanism is complex and need to be verified through psychophysical experiments. We proposed a novel haptic platform coupled with kinesthetic and tactile to render the macro texture. The StimTac is used as the end effector for both fine texture perception and curvature perception. As the texture perception of StimTac based on friction reduction by means of squeeze film effect was well studied, we focus on in this paper the perception of the profile of macro texture. Three motion models are analyzed: the basic motion model, the velocity compensation model and the position compensation model.

The future work is to verify the motion models and the perception effect coupled of fine texture and curvature. In addition, the deformation of fingertip's tissue, the force applied by the finger, the shearing force fed back by the surface, the friction force between the finger and the surface are need to be calibrated through psychophysical experiments, such as the perception match experiment, the just noticeable difference experiment, etc.

\section{Acknowledgment}

This study was supported by the National Nature Science Foundations of China (No.61403320), and the open funding project of State Key Laboratory of Virtual Reality Tech-nology and Systems, Beihang University (Grant No. BUAA-VR-15KF-01).The authors want to thank the group of Prof. Betty Lemaire-Semail at University of Lille and L2EP laboratory, France, for the loan of StimTac device.

\section{References}

1. V. Hayward, Yi, D, Change of height: An approach to the haptic display of shape and texture without surface normal. In: Experimental Robotics VIII, B. Siciliano, P. Dario (eds.), Springer Tracts in Advanced Robotics, Springer, Heidelberg, pp. 570-579, (2003)

2. R. Vitushinsky,S.Schmitz,A.Ludwig,Bistablethin-film shape memory actuators for applications in tactile displays, J. Micro electro mechanical Systems, 18(1), pp. 186-194, (2009)

3. G. H. Yang, K. U.Kyung, M. A. Srinivasan, D. S. Kwon, Development of quantitative tactile display device to provide both pin- array-type tactile feed back and thermal feed back. In: Second Joint Euro Haptics Conference and Symposium onHaptic Interfaces for Virtual Environment and Teleoperator Systems (WHC'07), pp. 578-579,(2007)

4. V. Hayward, Display of haptic shape at different scales. In: Proc. Eurohaptics 2004,Keynote paper, Munich Germany, pp. 20-27,(2004)

5. O. Bau, I. Poupyrev, A.Israr, C. Harrison, Tesla Touch: electrovibration for touch surfaces. In: Proc. 23th Ann. ACM Symp. User Interface Software and Technology (UIST'10),New York, USA, pp. 283-292,(2010)

6. M. Biet, F. Giraud, B. Lemaire-Semail, Squeeze film effect for the design of an ultrasonic tactile plate. IEEE Trans. Ultrasonics, Ferroelectrics and Frequency Control,54(12), pp. 2678-2688, (2007)

7. R. J. Webster, T.E. Murphy, L. N. Verner, A. M. Okamura, A novel two dimension altactile slip display: design, kinematics and perceptual experiments. ACM Trans. Applied Perception,2(2), pp. 150-165, (2005) 
8. G. Robles-De-La-Torre, V. Hayward, Force can overcome object geometry in the perception of shape through active touch, Nature, 412( 6845), pp. 445-448, (2007)

9. S. Choi, H. Z. an, Toward realistic haptic rendering of surface textures. IEEE Comput. Graph. Appl, 24(2), pp. 40-47, (2004)

10. M. Amberg, F. Giraud, B. Lemaire-Semail, STIMTAC, a tactile input device with programmable friction. In: Proc. UIST'11,SantaBarbara, CA, USA, pp.7-8.(2011)

11. T. Zeng, F. Giraud, B .Lemaire-Semail, M. Amberg, Contribution of slip cue to curvature perception through active and dynamic touch. IEEE Trans. Haptics,6(4), pp. 408-416, (2013)

12. S.C. Pont, A. M. L. Kappers, J. J. Koenderink, Similar mechanismsunderlie curvature comparison by static and by dynamic touch. Perception \& Psychophysics, 61(5), pp. 874-894, (1999)

13. M. W. A. Wijntjes, A. Sato, V. Hayward, A. M. L. Kappers, Local surface orientation dominates haptic curvature discrimination. IEEE Transactions on haptics,2(2), pp. 94-102, (2009)

14. L. Winfield, J. Glassmire, J. E. Colgate, M. Peshkin, T-pad: Tactile pattern display through variable friction reduction. In: Second Joint Euro Haptics Conference and Symposium on Haptic Interfaces for Virtual Environment and Teleoperator Systems (WHC'07),pp.421-426, (2007)

15. H. Kotani, M. Takasaki, T. Mizuno, Surface acoustic wave tactile display using a large size glass transducer. In: International Conference on Mechatronics and Automation, Harbin, pp. 198-203, (2007) 